\title{
Should we adhere to the new diagnostic recommendations for arterial hypertension?
}

\section{¿Debemos acogernos a las nuevas recomendaciones de diagnóstico sobre hipertensión arterial?}

\author{
Dagnóvar Aristizábal-Ocampo
}

\author{
SICOR Clinical and Research Center, Medellín, Colombia
}

Received 29 December 2017; accepted 29 December 2017

Available online 11 January 2018

Early disease detection and diagnostic precision have great benefits for public health; however, these gains come with a price. With greater evidence from clinical trials, the development of new diagnostic methods, and improved medical technologies, the line between what is normal and abnormal becomes very faint, and small physiological changes can turn our apparent normality into abnormality. This is the scene at which we have arrived in arterial hypertension as a result of the recent publication of the American College of Cardiology and American Heart Association (ACC/AHA ${ }^{1}$ joint guidelines, which consider a systolic arterial pressure $(A P) \geq 120 \mathrm{~mm} \mathrm{Hg}$ to be elevated, and classifies Stage I hypertension beginning at $130 / 80 \mathrm{~mm} \mathrm{Hg}$. This new classification contrasts with that published in the latest European hypertension guideline, ${ }^{2}$ and in the guideline termed JNC 8.

Arterial hypertension is one of the factors with the greatest population attributable risk (PAR). In the study carried out by our group in Medellín, Colombia, there was a $32 \%$ PAR for coronary disease when a $140 / 90 \mathrm{~mm} \mathrm{Hg}$ cutoff point was used for hypertension. In other words, $32 \%$ of coronary events (myocardial infarction, chest angina) would be neutralized if the referenced population were not hypertensive. ${ }^{4}$ Now, under the new cut-off points, this value 12.016

DOI of original article: http://dx.doi.org/10.1016/j.rccar.2017.

E-mail address: dagnovar@une.net.co would be $40 \%$. The World Health Organization researchers have found that systolic pressures over $115 \mathrm{~mm} \mathrm{Hg}$, or diastolic pressures over $75 \mathrm{~mm} \mathrm{Hg}$, explain $49 \%$ of the incidence of coronary events and $62 \%$ of cerebrovascular accidents, ${ }^{5}$ a fact which coincides with what was observed in our population.

The benefits of maintaining a normal AP, in terms of neutralizing cardiovascular risk, are undeniable. This, as we well know, can be achieved in most individuals through measures such as maintaining an appropriate weight, performing frequent physical activity, reducing the consumption of salt and alcohol, and increasing the consumption of fruits and vegetables. ${ }^{1,6}$ Therefore, the answer to the question "Is an AP $<120 / 80 \mathrm{~mm} \mathrm{Hg}$ considered to be normotensive?, is affirmative, and was already clear.

That said, the big change with these latest guidelines is the proposal that therapeutic intervention (nonpharmacological) should begin at pressures above $120 \mathrm{~mm}$ $\mathrm{Hg}$ if the cardiovascular risk is $<10 \%$ at 10 years, and if the systolic pressure is $130 \mathrm{~mm} \mathrm{Hg}$ and the risk is > $10 \%$, pharmacological treatment should also be included. They also indicate that in individuals who are already hypertensive, AP should be reduced not to $140 \mathrm{~mm} \mathrm{Hg}$, but rather to values < $130 \mathrm{~mm} \mathrm{Hg}$. In light of these modifications, I believe that the criteria for determining in which patients, and at what intensity, treatment should be carried out, need to be much better defined; not just the point to which AP is intended to be reduced. In other words, the management of hypertensive patients must be much more individualized. 
Ever since it became clear that a high AP had a direct relationship with cardiovascular disease, we have questioned whether reducing AP more than what was published in previous guidelines (below $140 \mathrm{~mm} \mathrm{Hg}$ ) provides greater cardiovascular benefits. This has been a research topic over the last three decades, and because of these findings, the ACC 2017 guideline declarations do not take us by surprise. With the SPRINT ${ }^{7}$ and ACCORD $^{8}$ studies, there are more than 20 clinical studies, with 54,000 patients included, which show that a more intense hypertension treatment is better than a less intense one for several high risk groups. ${ }^{9}$

The SPRINT ${ }^{7}$ results agree with some recent metaanalyses which have included several clinical trials with a large number of patents and outcomes. Xie et al. ${ }^{10}$ compared the effect of intensive treatment versus less intensive treatment for lowering pressure, and the effects on cardiovascular and kidney outcomes. The findings show that intensive treatment achieved a lower systolic pressure at $133 \mathrm{~mm} \mathrm{Hg}$, compared with $140 \mathrm{~mm} \mathrm{Hg}$ in the less intensive treatment group. This difference had an associated significant reduction in the risk of major cardiovascular events, myocardial infarction and cerebrovascular accidents (a relative risk reduction of 15-20\%); and, in diabetic patients, a significant reduction in albuminuria or retinopathy progression. These beneficial effects should be contrasted with the number of adverse side effects which have been regularly associated with treatment carried to lower pressures. In SPRINT, patients treated with a pressure goal of $120 \mathrm{~mm} \mathrm{Hg}$ had more hypotension, syncope, and electrolyte disorders, as well as kidney damage and failure.

While these trials support a change in the antihypertensive treatment AP reduction goal, they also indicate the need for a careful selection of in whom, and how, this AP reduction should be carried out. Given that the therapeutic effect is differential, depending on the level of cardiovascular risk and the type of patient being considered (the elderly, diabetics, those with systolic hypertension, those with kidney disease, etc.), more studies are needed which better characterize hypertensives according to their phenotypical features, other risk markers, and comorbidities, in order to determine the type of treatment to select. ${ }^{11}$ Again, this is a call to individualize treatment according to the degree of risk and comorbidities.

\section{Treatment selection should take into account the type of patient and the quantified cardiovascular risk}

Not all antihypertensives, nor all types of pharmacological combinations, reduce AP in the same way. Neither is cardiovascular protection achieved in all hypertensives with the lowering of arterial pressures, if potential adverse effects are not taken into account. This clearly leads to the categorization of risk being a central factor in decision making, to determine the intensity of pharmacological treatment and the treatment goal, in terms of AP. The recent 2017 ACC guideline states it like this: "for a given amount of reduction in AP with hypertensives, a smaller number of high risk individuals need to be treated to prevent a cardiovascular event, compared with low risk individuals"'. ${ }^{1}$ For example, a diabetic patient, or one with chronic kidney disease requires a more intensive treatment strategy than a young patient, or one who does not have other identified risks. Likewise, patients with arterial rigidity or with $a \geq$ $10 \%$ risk for atherosclerosis at 10 years should be included in this higher risk group. Hypertensive patients with coronary disease who require secondary prevention, and in whom achieving a $120 \mathrm{~mm} \mathrm{Hg}$ AP would often be accompanied by an excessive reduction in diastolic pressure, are also part of this group. It is in these patients in whom the combination of non-pharmacological measures plus pharmacological treatment should be applied, effectively and skillfully. And । must emphasize ëffectivelÿ, since the prescription of pharmacological and non-pharmacological treatment by the clinician is just one part of the plan resulting in benefits for the patient. The proper execution of this therapeutic plan requires patient education and support by an interdisciplinary team.

\section{Important considerations for implementing an antihypertensive treatment with stringent goals}

Accepting the evidence which shows that a stricter AP goal provides better cardiovascular protection, once the treatment has been individualized to mitigate potential adverse effects, the following elements should be taken into account:

1. The potential benefit vs. adverse effects of intensive treatment: it is very important to establish the level of risk and the comorbidities to be addressed, and to consider if the therapeutic goal might cause risks which could affect the expected benefit. If the cardiovascular risk is greater than $10 \%$ at 10 years, the intervention to lead a patient to a goal of $130 \mathrm{~mm} \mathrm{Hg}$ or less will require an intensive pharmacological management with up to three or more medications. This treatment must take into account that: a) antihypertensives have additive effects and, in general, using lower doses and adding a new class of antihypertensives (combination therapy) achieves a greater decrease in AP than doubling the dose of a single medication (this is true when using thiazides, beta-blockers, angiotensin ॥ receptor antagonists, and calcium channel blockers ${ }^{12}$ ); and b) achieving a systolic pressure of $120-130 \mathrm{~mm} \mathrm{Hg}$ in most patients > 75 will often be accompanied by a diastolic pressure $<70 \mathrm{~mm} \mathrm{Hg}$, and this can compromise coronary flow, along with that of other territories, due to the known diastolic pressure J-curve. ${ }^{13}$

2. The way in which medications interact in combination should be well understood when two or three antihypertensives are needed to achieve these goals. This aspect is even more important due to the effects of intense AP reduction on kidney function. Both in the SPRINT and the ACCORD studies, a greater reduction in AP was accompanied by an increase in creatinine levels, and even acute kidney failure. This, together with the electrolyte disturbances more common in this intensely treated group, means one lesion or acute kidney failure event for every 63 patients treated, one patient developing hypotension 
for every 99 patients treated, and one electrolyte disturbance for every 128 patients treated. ${ }^{14}$

3. The new hypertensive label for previously normotensive patients: in light of the categorization of high pressure beginning at $120 \mathrm{~mm} \mathrm{Hg}$, it is fitting for clinicians to ask themselves how to avoid making individuals feel labeled and ill, knowing they have been told that they have high blood pressure. This is a challenge in the implementation of this new standard which has led to many who previously had enjoyed being normotensive, now having an abnormal pressure. Certainly, what has come to be called the "tyranny of normality" must be considered in this new scenario.

4. The epidemiological and health system consequences: । have avoided referring to the effects of this new classification on public health in Colombia and other Latin American countries, in terms of the increased diagnosis and treatment of hypertension. However, having $25 \%$ more hypertensives in the population will bring unavoidable consequences to our health systems, most of which are based on public insurance, and which, even with the current prevalence, poorly control the hypertension in our populations. The effects of this new "burden", of people with high blood pressure, and of hypertensives who require intensive treatment, will have a strong impact on our health systems' resources.

\section{References}

1. Whelton PK, Carey RM, Aronow WS, Casey DE, Jr., Collins KJ, Dennison Himmelfarb C et al., 2017. ACC/AHA/AAPA/ABC/ACPM/AGS/APhA/ASH/ASPC/NMA/PCNA

Guideline for the Prevention, Detection, Evaluation, and Management of High Blood Pressure in Adults: A Report of the American College of Cardiology/American Heart Association Task Force on Clinical Practice Guidelines. J Am Coll Cardiol. 2017, 23976;. 10.1161/HYP.0000000000000066

2. Mancia G, Fagard R, Narkiewicz K, Redon J, Zanchetti A, Bohm M, et al. 2013 ESH/ESC guidelines for the management of arterial hypertension: the Task Force for the Management of Arterial Hypertension of the European Society of Hypertension (ESH) and of the European Society of Cardiology (ESC). Eur Heart J. 2013;34:2159-219.
3. James PA, Oparil S, Carter BL, Cushman WC, DennisonHimmelfarb C, Handler J, et al. 2014 evidence-based guideline for the management of high blood pressure in adults: report from the panel members appointed to the Eighth Joint National Committee (JNC 8). JAMA. 2014;311:507-20.

4. Torres Y, Aristizabal D, Montoya L. Diagnóstico del riesgo cardiovascular global, evaluación de su impacto poblacional, Medellín y sus corregimientos 2007-2008. Medellín 2008. Disponible en: http: / / www. medellin.gov.co/irj/go/ km/docs/AlcaldiaMedellin /SecretariaSalud/SitioTemporalSalud/descargables/EstudiosElnvestigaciones/Diagnostico_\%20riesgo_\%20cardiovascular Medellin_\%202007_2008.pdf

5. Brundtland GH. From the World Health Organization Reducing risks to health, promoting healthy life. JAMA. 2002;288:1974.

6. Hedayati SS, Elsayed EF, Reilly RF. Non-pharmacological aspects of blood pressure management: what are the data? Kidney Int. 2011;79:1061-70.

7. Group SR, Wright JT Jr, Williamson JD, Whelton PK, Snyder JK, Sink KM, et al. A randomized trial of intensive versus standard blood-pressure control. N Engl J Med. 2015;373:2103-16.

8. Group AS, Cushman WC, Evans GW, Byington RP, Goff DC Jr, Grimm RH Jr, et al. Effects of intensive blood-pressure control in type 2 diabetes mellitus. N Engl J Med. 2010;362:1575-85.

9. Atkins ER, Rodgers A. More versus less blood pressure lowering: an update. Clin Ther. 2016;38:2135-41.

10. Xie X, Atkins E, Lv J, Bennett A, Neal B, Ninomiya T, et al. Effects of intensive blood pressure lowering on cardiovascular and renal outcomes: updated systematic review and metaanalysis. Lancet. 2016;387(10017):435-43.

11. Agabiti Rosei E. Target blood pressure for treatment: should current recommendations be changed? Hypertension. 2016;68:263-5.

12. Wald DS, Law M, Morris JK, Bestwick JP, Wald NJ. Combination therapy versus monotherapy in reducing blood pressure: meta-analysis on 11,000 participants from 42 trials. Am J Med. 2009; 122:290-300.

13. Mancia G, Grassi G. Aggressive blood pressure lowering is dangerous: the J-curve: pro side of the arguement. Hypertension. 2014;63:29-36.

14. Bress AP, Kramer H, Khatib R, Beddhu S, Cheung AK, Hess R, et al. Potential Deaths Averted and Serious Adverse Events Incurred From Adoption of the SPRINT (Systolic Blood Pressure Intervention Trial) Intensive Blood Pressure Regimen in the United States: Projections From NHANES (National Health and Nutrition Examination Survey). Circulation. 2017;135: $1617-28$. 